General Mathematics Vol. 28, No. 1 (2020), 25-39

DOI: $10.2478 / \mathrm{gm}-2020-0003$

S sciendo

\title{
Some Hadamard and Simpson type inequalities via $s$-convexity and their applications ${ }^{1}$
}

\author{
Musa Çakmak, Mevlüt Tunç
}

\begin{abstract}
In this study, the authors establish and generalize some inequalities of Hadamard and Simpson type based on $s$-convexity in the second sense. Some applications are also given and generalized. Examples are given to show the results. The results generalize the integral inequalities in articles of Sarikaya and $\mathrm{Xi}$.
\end{abstract}

2010 Mathematics Subject Classification: 26D07, $26 \mathrm{D} 15$.

Key words and phrases: $s$-convex functions, Hadamard inequality, Simpson inequality, Bullen inequality.

\section{Introduction}

To establish analytic inequalities, one of the most efficient way is the property of convexity of a dedicated function. Notedly, in the theory of higher transcendental functions, there are many significant applications. We can use the integral inequalities in order to study qualitative and quantitative properties of integrals (see $[9,10,13])$. Thing continuing to bewilder us by indicating new inferences, new difficulties and also new open questions are a major mathematical outcome.

The Hermite-Hadamard inequality: Let $\Psi: J \subseteq \mathbb{R} \rightarrow \mathbb{R}$ be a convex function and $a_{1}, a_{2} \in J$ with $a_{1}<a_{2}$.

$$
\Psi\left(\frac{a_{1}+a_{2}}{2}\right) \leq \frac{1}{a_{2}-a_{1}} \int_{a_{1}}^{a_{2}} \Psi(\varkappa) d \varkappa \leq \frac{\Psi\left(a_{1}\right)+\Psi\left(a_{2}\right)}{2}
$$

\footnotetext{
${ }^{1}$ Received 15 February, 2019

Accepted for publication (in revised form) 16 February, 2020
} 
If $\Psi$ is concave, this double inequality hold in the inverse way. See $[3,10]$ for details. The Simpson inequality:

$$
\begin{aligned}
& \left|\frac{1}{3}\left[\frac{\Psi\left(a_{1}\right)+\Psi\left(a_{2}\right)}{2}+2 \Psi\left(\frac{a_{1}+a_{2}}{2}\right)\right]-\frac{1}{a_{2}-a_{1}} \int_{a_{1}}^{a_{2}} \Psi(x) d \varkappa\right| \\
\leq & \frac{1}{1280}\left\|\Psi^{(4)}\right\|_{\infty}\left(a_{2}-a_{1}\right)^{4},
\end{aligned}
$$

where the mapping $\Psi:\left[a_{1}, a_{2}\right] \rightarrow \mathbb{R}$ is assumed to be four times continuously differentiable on the interval and $\Psi^{(4)}$ to be bounded on $\left(a_{1}, a_{2}\right)$, that is, $\left\|\Psi^{(4)}\right\|_{\infty}=$ $\sup _{t \in\left(a_{1}, a_{2}\right)}\left|\Psi^{(4)}(t)\right|<\infty$. See $[1,4,11,12]$ for details.

The Bullen inequality:

$$
\frac{1}{a_{2}-a_{1}} \int_{a_{1}}^{a_{2}} \Psi(\varkappa) d \varkappa \leq \frac{1}{2}\left[\frac{\Psi\left(a_{1}\right)+\Psi\left(a_{2}\right)}{2}+\Psi\left(\frac{a_{1}+a_{2}}{2}\right)\right],
$$

provided that $\Psi:\left[a_{1}, a_{2}\right] \rightarrow \mathbb{R}$ is a convex function on $\left[a_{1}, a_{2}\right]$. (see for example $[5$, p.10]) for details.

In [7], Hudzik and Maligranda considered among others the class of functions which are $s$-convex in the second sense. This class is defined in the following way: a function $\Psi: \mathbb{R}_{+} \rightarrow \mathbb{R}$, where $\mathbb{R}_{+}=[0, \infty)$, is said to be $s$-convex in the second sense if

$$
\Psi(\alpha \lambda+(1-\alpha) \mu) \leq \alpha^{s} \Psi(\lambda)+(1-\alpha)^{s} \Psi(\mu)
$$

for all $\lambda, \mu \in[0, \infty), \alpha \in[0,1]$ and for some fixed $s \in(0,1]$. This class of $s$ convex functions is usually denoted by $K_{s}^{2}$. It can be smoothly seen that for $s=1$, $s$-convexity reduces to the ordinary convexity of functions defined on $[0, \infty)$.

Recently, in [11, 12], Sarikaya et al. presented the important integral identity including the first-order derivative of $\Psi$ to establish many interesting Simpson-type inequalities for convex and $s$-convex functions.

Meanwhile, in [14], Xi et al. presented the following two important integral identities including the first-order derivatives to establish many interesting HermiteHadamard-type inequalities for convex functions.

In this study, some new Hadamard and Simpson type integral inequalities for differentiable functions are established, and are applied to produce some inequalities of special means. Examples are given to show the results. The results generalize the integral inequalities in [12] and [14].

\section{Main results for $s$-convex functions}

To demonstrate our main results, we need the following lemmas that have been derived in [14]: 
Lemma 1 [14] Let $\Psi: J \subseteq \mathbb{R} \rightarrow \mathbb{R}$ be differentiable function on $J^{\circ}, a_{1}, a_{2} \in J$, with $a_{1}<a_{2}$. If $\Psi^{\prime} \in L\left[a_{1}, a_{2}\right]$ and $\lambda, \mu \in \mathbb{R}$ then

$(4)$

$$
\begin{aligned}
& \frac{\lambda \Psi\left(a_{1}\right)+\mu \Psi\left(a_{2}\right)}{2}+\frac{2-\lambda-\mu}{2} \Psi\left(\frac{a_{1}+a_{2}}{2}\right)-\frac{1}{a_{2}-a_{1}} \int_{a_{1}}^{a_{2}} \Psi(\varkappa) d \varkappa \\
= & \frac{a_{2}-a_{1}}{4} \int_{0}^{1}\left[(1-\lambda-\alpha) \Psi^{\prime}\left(\alpha a_{1}+(1-\alpha) \frac{a_{1}+a_{2}}{2}\right)\right. \\
& \left.+(\mu-\alpha) \Psi^{\prime}\left(\alpha \frac{a_{1}+a_{2}}{2}+(1-\alpha) a_{2}\right)\right] d \alpha
\end{aligned}
$$

Lemma 2 [14]For $\varkappa>0$ and $0 \leq \gamma \leq 1$, one has

$$
\begin{aligned}
\int_{0}^{1}|\gamma-\alpha|^{\varkappa} d \alpha & =\frac{\gamma^{\varkappa+1}+(1-\gamma)^{\varkappa+1}}{\varkappa+1} \\
\int_{0}^{1} \alpha|\gamma-\alpha|^{\varkappa} d \alpha & =\frac{\gamma^{\varkappa+2}+(\varkappa+1+\gamma)(1-\gamma)^{\varkappa+1}}{(\varkappa+1)(\varkappa+2)} .
\end{aligned}
$$

Theorem 1 Let $\Psi: J \subseteq \mathbb{R} \rightarrow \mathbb{R}$ be differentiable function on $J^{\circ}, a_{1}, a_{2} \in J, 0 \leq \lambda$, $\mu \leq 1$, and $\Psi^{\prime} \in L\left[a_{1}, a_{2}\right]$. If $\left|\Psi^{\prime}(\varkappa)\right|^{r}$ is s-convex function in the second sense on $\left[a_{1}, a_{2}\right]$ for some fixed $s \in(0,1], p, r>1,1 / p+1 / r=1$, then

$(6)$

$$
\begin{aligned}
& \left|\frac{\lambda \Psi\left(a_{1}\right)+\mu \Psi\left(a_{2}\right)}{2}+\frac{2-\lambda-\mu}{2} \Psi\left(\frac{a_{1}+a_{2}}{2}\right)-\frac{1}{a_{2}-a_{1}} \int_{a_{1}}^{a_{2}} \Psi(\varkappa) d \varkappa\right| \\
\leq & \frac{a_{2}-a_{1}}{4}\left(\frac{(1-\lambda)^{p+1}+\lambda^{p+1}}{p+1}\right)^{\frac{1}{p}} \\
& \times\left(\frac{2^{s+1}-1}{2^{s}(s+1)}\left|\Psi^{\prime}\left(a_{1}\right)\right|^{r}+\frac{1}{2^{s}(s+1)}\left|\Psi^{\prime}\left(a_{2}\right)\right|^{r}\right)^{\frac{1}{r}} \\
& +\frac{a_{2}-a_{1}}{4}\left(\frac{\mu^{p+1}+(1-\mu)^{p+1}}{p+1}\right)^{\frac{1}{p}} \\
& \times\left(\frac{1}{2^{s}(s+1)}\left|\Psi^{\prime}\left(a_{1}\right)\right|^{r}+\frac{2^{s+1}-1}{2^{s}(s+1)}\left|\Psi^{\prime}\left(a_{2}\right)\right|^{r}\right)^{\frac{1}{r}} .
\end{aligned}
$$

Proof. Assume that $p>1$, by Lemma 1 and using the well known Hölder 
inequality, we have

$$
\begin{aligned}
& \left|\frac{\lambda \Psi\left(a_{1}\right)+\mu \Psi\left(a_{2}\right)}{2}+\frac{2-\lambda-\mu}{2} \Psi\left(\frac{a_{1}+a_{2}}{2}\right)-\frac{1}{a_{2}-a_{1}} \int_{a_{1}}^{a_{2}} \Psi(\varkappa) d \varkappa\right| \\
\leq & \frac{a_{2}-a_{1}}{4}\left[\int_{0}^{1}|1-\lambda-\alpha|\left|\Psi^{\prime}\left(\alpha a_{1}+(1-\alpha) \frac{a_{1}+a_{2}}{2}\right)\right| d \alpha\right. \\
& \left.+\int_{0}^{1}|\mu-\alpha|\left|\Psi^{\prime}\left(\alpha \frac{a_{1}+a_{2}}{2}+(1-\alpha) a_{2}\right)\right| d \alpha\right] \\
\leq & \frac{a_{2}-a_{1}}{4}\left(\int_{0}^{1}|1-\lambda-\alpha|^{p} d t\right)^{\frac{1}{p}}\left(\int_{0}^{1}\left|\Psi^{\prime}\left(\alpha a_{1}+(1-\alpha) \frac{a_{1}+a_{2}}{2}\right)\right|^{r} d \alpha\right)^{\frac{1}{r}} \\
& +\frac{a_{2}-a_{1}}{4}\left(\int_{0}^{1}|\mu-\alpha|^{p} d \alpha\right)^{\frac{1}{p}}\left(\int_{0}^{1}\left|\Psi^{\prime}\left(\alpha \frac{a_{1}+a_{2}}{2}+(1-\alpha) a_{2}\right)\right|^{r} d \alpha\right)^{\frac{1}{r}} .
\end{aligned}
$$

Since $\left|\Psi^{\prime}(\varkappa)\right|^{r}$ is $s$-convex on $\left[a_{1}, a_{2}\right]$, then we get

$$
\begin{aligned}
& \int_{0}^{1}\left|\Psi^{\prime}\left(\alpha a_{1}+(1-\alpha) \frac{a_{1}+a_{2}}{2}\right)\right|^{r} d \alpha \\
\leq & \int_{0}^{1}\left(\left(\frac{1+\alpha}{2}\right)^{s}\left|\Psi^{\prime}\left(a_{1}\right)\right|^{r}+\left(\frac{1-\alpha}{2}\right)^{s}\left|\Psi^{\prime}\left(a_{2}\right)\right|^{r}\right) d \alpha \\
= & \frac{2^{s+1}-1}{2^{s}(s+1)}\left|\Psi^{\prime}\left(a_{1}\right)\right|^{r}+\frac{1}{2^{s}(s+1)}\left|\Psi^{\prime}\left(a_{2}\right)\right|^{r}
\end{aligned}
$$

and

$$
\begin{aligned}
& \int_{0}^{1}\left|\Psi^{\prime}\left(\alpha \frac{a_{1}+a_{2}}{2}+(1-\alpha) a_{2}\right)\right|^{r} d \alpha \\
\leq & \int_{0}^{1}\left(\left(\frac{\alpha}{2}\right)^{s}\left|\Psi^{\prime}\left(a_{1}\right)\right|^{r}+\left(\frac{2-\alpha}{2}\right)^{s}\left|\Psi^{\prime}\left(a_{2}\right)\right|^{r}\right) d \alpha \\
= & \frac{1}{2^{s}(s+1)}\left|\Psi^{\prime}\left(a_{1}\right)\right|^{r}+\frac{2^{s+1}-1}{2^{s}(s+1)}\left|\Psi^{\prime}\left(a_{2}\right)\right|^{r}
\end{aligned}
$$

where we have used the fact that

$$
\int_{0}^{1}|1-\lambda-\alpha|^{p} d \alpha=\frac{(1-\lambda)^{p+1}+\lambda^{p+1}}{p+1}
$$

and

$$
\int_{0}^{1}|\mu-\alpha|^{p} d \alpha=\frac{\mu^{p+1}+(1-\mu)^{p+1}}{p+1} .
$$

This completes the proof.

If taking $\lambda=\mu$ in Theorem 1, the following corollary derived. 
Corollary 1 Let $\Psi: J \subseteq \mathbb{R} \rightarrow \mathbb{R}$ be differentiable function on $J^{\circ}$, $a_{1}, a_{2} \in J$, $0 \leq \lambda \leq 1$, and $\Psi^{\prime} \in L\left[a_{1}, a_{2}\right]$. If $\left|\Psi^{\prime}(\varkappa)\right|^{r}$ is s-convex function on $\left[a_{1}, a_{2}\right]$ for some fixed $s \in(0,1]$, and $1 / p+1 / r=1$, then

$$
\begin{aligned}
& \quad\left|\lambda \frac{\Psi\left(a_{1}\right)+\Psi\left(a_{2}\right)}{2}+(1-\lambda) \Psi\left(\frac{a_{1}+a_{2}}{2}\right)-\frac{1}{a_{2}-a_{1}} \int_{a_{1}}^{a_{2}} \Psi(\varkappa) d \varkappa\right| \\
& \leq \frac{a_{2}-a_{1}}{4}\left(\frac{(1-\lambda)^{p+1}+\lambda^{p+1}}{p+1}\right)^{\frac{1}{p}} \\
& \quad \times\left\{\left(\frac{2^{s+1}-1}{2^{s}(s+1)}\left|\Psi^{\prime}\left(a_{1}\right)\right|^{r}+\frac{1}{2^{s}(s+1)}\left|\Psi^{\prime}\left(a_{2}\right)\right|^{r}\right)^{\frac{1}{r}}\right. \\
& \left.+\left(\frac{1}{2^{s}(s+1)}\left|\Psi^{\prime}\left(a_{1}\right)\right|^{r}+\frac{2^{s+1}-1}{2^{s}(s+1)}\left|\Psi^{\prime}\left(a_{2}\right)\right|^{r}\right)^{\frac{1}{r}}\right\}
\end{aligned}
$$

Remark 1 In Theorem 1, if we take $\lambda=\mu=1 / 3$, then Theorem 1 reduces to [12, Theorem 9]. Hence, generalizations of the results of Sarikaya et al. in [12]. is the resulted in Theorem 1.

If we take $s=1$ and $\lambda=\mu=1 / 2,2 / 3,1 / 3$, respectively, in Theorem 1 , the following inequalities can be deduced.

Corollary 2 Let $\Psi: J \subseteq \mathbb{R} \rightarrow \mathbb{R}$ be a differentiable function on $J^{\circ}, a_{1}, a_{2} \in J$ with $a_{1}<a_{2}$, and $\Psi^{\prime} \in L\left[a_{1}, a_{2}\right]$. If $\left|\Psi^{\prime}(\varkappa)\right|^{r}$ is convex function on $\left[a_{1}, a_{2}\right]$ for $1 / p+1 / r=1$, then

$$
\begin{aligned}
& \left|\frac{1}{2}\left[\frac{\Psi\left(a_{1}\right)+\Psi\left(a_{2}\right)}{2}+\Psi\left(\frac{a_{1}+a_{2}}{2}\right)\right]-\frac{1}{a_{2}-a_{1}} \int_{a_{1}}^{a_{2}} \Psi(\varkappa) d \varkappa\right| \\
\leq & \frac{a_{2}-a_{1}}{8(p+1)^{1 / p} 4^{1 / r}} \\
& \times\left[\left(3\left|\Psi^{\prime}\left(a_{1}\right)\right|^{r}+\left|\Psi^{\prime}\left(a_{2}\right)\right|^{r}\right)^{\frac{1}{r}}+\left(\left|\Psi^{\prime}\left(a_{1}\right)\right|^{r}+3\left|\Psi^{\prime}\left(a_{2}\right)\right|^{r}\right)^{\frac{1}{r}}\right] \\
\leq & \frac{a_{2}-a_{1}}{4^{1+1 / r}}\left(\frac{\left(1+2^{p+1}\right)}{3^{p+1}(p+1)}\right)^{\frac{1}{p}}\left[\Psi\left(a_{1}\right)+\Psi\left(a_{2}\right)+\Psi\left(\frac{a_{1}+a_{2}}{2}\right)\right]-\frac{1}{a_{2}-a_{1}} \int_{a_{1}}^{a_{2}} \Psi(\varkappa) d \varkappa \mid \\
& \times\left[\left(3\left|\Psi^{\prime}\left(a_{1}\right)\right|^{r}+\left|\Psi^{\prime}\left(a_{2}\right)\right|^{r}\right)^{\frac{1}{r}}+\left(\left|\Psi^{\prime}\left(a_{1}\right)\right|^{r}+3\left|\Psi^{\prime}\left(a_{2}\right)\right|^{r}\right)^{\frac{1}{r}}\right]
\end{aligned}
$$


$(7)$

$$
\begin{aligned}
& \left|\frac{1}{6}\left[\Psi\left(a_{1}\right)+\Psi\left(a_{2}\right)+4 f\left(\frac{a_{1}+a_{2}}{2}\right)\right]-\frac{1}{a_{2}-a_{1}} \int_{a_{1}}^{a_{2}} \Psi(\varkappa) d \varkappa\right| \\
\leq & \frac{a_{2}-a_{1}}{4^{1+1 / r}}\left(\frac{\left(1+2^{p+1}\right)}{3^{p+1}(p+1)}\right)^{\frac{1}{p}} \\
& \times\left[\left(3\left|\Psi^{\prime}\left(a_{1}\right)\right|^{r}+\left|\Psi^{\prime}\left(a_{2}\right)\right|^{r}\right)^{\frac{1}{r}}+\left(\left|\Psi^{\prime}\left(a_{1}\right)\right|^{r}+3\left|\Psi^{\prime}\left(a_{2}\right)\right|^{r}\right)^{\frac{1}{r}}\right]
\end{aligned}
$$

If taking $\lambda=\mu=1 / 2$, in Theorem 1 , the following inequalities can be deduced.

Corollary 3 Let $\Psi: J \subseteq \mathbb{R} \rightarrow \mathbb{R}$ be a differentiable function on $J^{\circ}, a_{1}, a_{2} \in J$, and $\Psi^{\prime} \in L\left[a_{1}, a_{2}\right]$. If $\left|\Psi^{\prime}(\varkappa)\right|^{r}$ is s-convex function on $\left[a_{1}, a_{2}\right]$ for some fixed $s \in(0,1]$, and $1 / p+1 / r=1$ and

$$
\frac{\Psi\left(a_{1}\right)+\Psi\left(a_{2}\right)}{2}=\Psi\left(\frac{a_{1}+a_{2}}{2}\right)
$$

then

$$
\begin{aligned}
& \left|\frac{\Psi\left(a_{1}\right)+\Psi\left(a_{2}\right)}{2}-\frac{1}{a_{2}-a_{1}} \int_{a_{1}}^{a_{2}} \Psi(\varkappa) d \varkappa\right| \\
= & \left|\Psi\left(\frac{a_{1}+a_{2}}{2}\right)-\frac{1}{a_{2}-a_{1}} \int_{a_{1}}^{a_{2}} \Psi(\varkappa) d \varkappa\right| \\
\leq & \frac{a_{2}-a_{1}}{8(p+1)^{\frac{1}{p}}}\left[\left(\frac{2^{s+1}-1}{2^{s}(s+1)}\left|\Psi^{\prime}\left(a_{1}\right)\right|^{r}+\frac{1}{2^{s}(s+1)}\left|\Psi^{\prime}\left(a_{2}\right)\right|^{r}\right)^{\frac{1}{r}}\right. \\
& \left.+\left(\frac{1}{2^{s}(s+1)}\left|\Psi^{\prime}\left(a_{1}\right)\right|^{r}+\frac{2^{s+1}-1}{2^{s}(s+1)}\left|\Psi^{\prime}\left(a_{2}\right)\right|^{r}\right)^{\frac{1}{r}}\right]
\end{aligned}
$$

Corollary 4 In Theorem 1, when $s, \lambda, \mu, p, r$ are taken like in the following, we obtain

i) For $s=0.3, \lambda=0.3, \mu=0.3, p=2, r=2$ in (6),

$$
\begin{aligned}
& \left|\frac{3}{10} \frac{\Psi\left(a_{1}\right)+\Psi\left(a_{2}\right)}{2}+\frac{14}{20} \Psi\left(\frac{a_{1}+a_{2}}{2}\right)-\frac{1}{b-a_{1}} \int_{a_{1}}^{a_{2}} \Psi(\varkappa) d \varkappa\right| \\
\leq & \frac{0.351\left(a_{2}-a_{1}\right)}{4}\left[\left(0.914\left|\Psi^{\prime}\left(a_{1}\right)\right|^{2}+0.625\left|\Psi^{\prime}\left(a_{2}\right)\right|^{2}\right)^{\frac{1}{2}}\right. \\
& \left.+\left(0.625\left|\Psi^{\prime}\left(a_{1}\right)\right|^{r}+0.914\left|\Psi^{\prime}\left(a_{2}\right)\right|^{2}\right)^{\frac{1}{2}}\right],
\end{aligned}
$$


ii) Taking $s=0.5, \lambda=0.5, \mu=0.5, p=2, r=2$ in (6) gives;

$$
\begin{aligned}
& \left|\frac{1}{2}\left[\frac{\Psi\left(a_{1}\right)+\Psi\left(a_{2}\right)}{2}+\Psi\left(\frac{a_{1}+a_{2}}{2}\right)\right]-\frac{1}{a_{2}-a_{1}} \int_{a_{1}}^{a_{2}} \Psi(\varkappa) d \varkappa\right| \\
\leq & \frac{0.289\left(a_{2}-a_{1}\right)}{4}\left[\left(0.862\left|\Psi^{\prime}\left(a_{1}\right)\right|^{2}+0.471\left|\Psi^{\prime}\left(a_{2}\right)\right|^{2}\right)^{\frac{1}{2}}\right. \\
& \left.+\left(0.471\left|\Psi^{\prime}\left(a_{1}\right)\right|^{2}+0.862\left|\Psi^{\prime}\left(a_{2}\right)\right|^{2}\right)^{\frac{1}{2}}\right],
\end{aligned}
$$

iii) Taking $s=0.75, \lambda=0.3, \mu=0.7, p=10, r=10 / 9$ in (6) gives;

$$
\begin{aligned}
& \left|\frac{3 \Psi\left(a_{1}\right)+7 \Psi\left(a_{2}\right)}{20}+\frac{1}{2} \Psi\left(\frac{a_{1}+a_{2}}{2}\right)-\frac{1}{a_{2}-a_{1}} \int_{a_{1}}^{a_{2}} \Psi(\varkappa) d \varkappa\right| \\
\leq & \frac{0.531\left(a_{2}-a_{1}\right)}{4}\left[\left(0.803\left|\Psi^{\prime}\left(a_{1}\right)\right|^{10 / 9}+0.34\left|\Psi^{\prime}\left(a_{2}\right)\right|^{10 / 9}\right)^{\frac{9}{10}}\right. \\
& \left.+\left(0.34\left|\Psi^{\prime}\left(a_{1}\right)\right|^{10 / 9}+0.803\left|\Psi^{\prime}\left(a_{2}\right)\right|^{10 / 9}\right)^{\frac{9}{10}}\right],
\end{aligned}
$$

iv) Taking $s=0.4, \lambda=0.2, \mu=0.8, p=3, r=3 / 2$ in (6) gives;

$$
\begin{aligned}
& \left|\frac{\Psi\left(a_{1}\right)+4 f\left(a_{2}\right)}{10}+\frac{1}{2} \Psi\left(\frac{a_{1}+a_{2}}{2}\right)-\frac{1}{a_{2}-a_{1}} \int_{a_{1}}^{a_{2}} \Psi(\varkappa) d \varkappa\right| \\
\leq & \frac{0.468\left(a_{2}-a_{1}\right)}{4}\left[\left(0.887\left|\Psi^{\prime}\left(a_{1}\right)\right|^{3 / 2}+0.541\left|\Psi^{\prime}\left(a_{2}\right)\right|^{3 / 2}\right)^{\frac{2}{3}}\right. \\
& \left.+\left(0.541\left|\Psi^{\prime}\left(a_{1}\right)\right|^{3 / 2}+0.887\left|\Psi^{\prime}\left(a_{2}\right)\right|^{3 / 2}\right)^{\frac{2}{3}}\right],
\end{aligned}
$$

v) Taking $s=0.4, \lambda=0.2, \mu=0.8, p=e, r=e /(e-1)$ in (6) gives;

$$
\begin{aligned}
& \left|\frac{\Psi\left(a_{1}\right)+4 f\left(a_{2}\right)}{10}+\frac{1}{2} \Psi\left(\frac{a_{1}+a_{2}}{2}\right)-\frac{1}{a_{2}-a_{1}} \int_{a_{1}}^{a_{2}} \Psi(\varkappa) d \varkappa\right| \\
\leq & \frac{0.455\left(a_{2}-a_{1}\right)}{4}\left[\left(0.887\left|\Psi^{\prime}\left(a_{1}\right)\right|^{e /(e-1)}+0.541\left|\Psi^{\prime}\left(a_{2}\right)\right|^{e /(e-1)}\right)^{\frac{e}{e-1}}\right. \\
& \left.+\left(0.541\left|\Psi^{\prime}\left(a_{1}\right)\right|^{e /(e-1)}+0.887\left|\Psi^{\prime}\left(a_{2}\right)\right|^{e /(e-1)}\right)^{\frac{e}{e-1}}\right]
\end{aligned}
$$

vi) Taking $s=1, \lambda=1 / 3, \mu=2 / 3, p=r=2$ in (6) gives;

$$
\begin{aligned}
& \left|\frac{\Psi\left(a_{1}\right)+2 f\left(a_{2}\right)}{6}+\frac{1}{2} \Psi\left(\frac{a_{1}+a_{2}}{2}\right)-\frac{1}{a_{2}-a_{1}} \int_{a_{1}}^{a_{2}} \Psi(\varkappa) d \varkappa\right| \leq \frac{a_{2}-a_{1}}{12} \\
& \times\left[\left(\frac{3}{4}\left|\Psi^{\prime}\left(a_{1}\right)\right|^{2}+\frac{1}{4}\left|\Psi^{\prime}\left(a_{2}\right)\right|^{2}\right)^{\frac{1}{2}}+\left(\frac{1}{4}\left|\Psi^{\prime}\left(a_{1}\right)\right|^{2}+\frac{3}{4}\left|\Psi^{\prime}\left(a_{2}\right)\right|^{2}\right)^{\frac{1}{2}}\right]
\end{aligned}
$$

etc. 
Theorem 2 Let $\Psi: J \subseteq \mathbb{R} \rightarrow \mathbb{R}$ be differentiable function on $J^{\circ}, a_{1}, a_{2} \in J, 0 \leq \lambda$, $\mu \leq 1$, and $\Psi^{\prime} \in L\left[a_{1}, a_{2}\right]$. If $\left|\Psi^{\prime}(\varkappa)\right|^{r}$ is s-convex function in the second sense on $\left[a_{1}, a_{2}\right]$ for $r \geq 1$, then

$$
\begin{aligned}
& \left|\frac{\lambda \Psi\left(a_{1}\right)+\mu \Psi\left(a_{2}\right)}{2}+\frac{2-\lambda-\mu}{2} \Psi\left(\frac{a_{1}+a_{2}}{2}\right)-\frac{1}{a_{2}-a_{1}} \int_{a_{1}}^{a_{2}} \Psi(\varkappa) d \varkappa\right| \\
(8) \leq & \frac{a_{2}-a_{1}}{8}\left(\frac{1}{2^{s-1}(s+1)(s+2)}\right)^{1 / r} \\
& \left\{\left(\left(2 \lambda^{2}-2 \lambda+1\right)\right)^{1-1 / r} \times\left[E\left|\Psi^{\prime}\left(a_{1}\right)\right|^{r}+L\left|\Psi^{\prime}\left(a_{2}\right)\right|^{r}\right]^{1 / r}\right. \\
& \left.+\left(\left(2 \mu^{2}-2 \mu+1\right)\right)^{1-1 / r} \times\left[J\left|\Psi^{\prime}\left(a_{1}\right)\right|^{r}+F\left|\Psi^{\prime}\left(a_{2}\right)\right|^{r}\right]^{1 / r}\right\}
\end{aligned}
$$

where

$$
\begin{aligned}
E & =\left(2(2-\lambda)^{s+2}+(\lambda-1)\left(s+2^{s+2}+2\right)+2^{s+1} s \lambda-1\right) \\
L & =\left(2 \lambda^{s+2}+s(1-\lambda)-2 \lambda+1\right) \\
J & =\left(2 \mu^{s+2}+s(1-\mu)-2 \mu+1\right) \\
F & =\left(2(2-\mu)^{s+2}+(\mu-1)\left(s+2^{s+2}+2\right)+2^{s+1} s \mu-1\right) .
\end{aligned}
$$

Proof. For $r \geq 1$, by using Lemma 1 , the $s$-convexity of $\left|\Psi^{\prime}(\varkappa)\right|^{r}$ on $\left[a_{1}, a_{2}\right]$ and the famous power mean inequality, we can hold

$$
\begin{aligned}
& \left|\frac{\lambda \Psi\left(a_{1}\right)+\mu \Psi\left(a_{2}\right)}{2}+\frac{2-\lambda-\mu}{2} \Psi\left(\frac{a_{1}+a_{2}}{2}\right)-\frac{1}{a_{2}-a_{1}} \int_{a_{1}}^{a_{2}} \Psi(\varkappa) d \varkappa\right| \\
\leq & \frac{a_{2}-a_{1}}{4}\left[\int_{0}^{1}|1-\lambda-\alpha|\left|\Psi^{\prime}\left(\alpha a_{1}+(1-\alpha) \frac{a_{1}+a_{2}}{2}\right)\right| d \alpha\right. \\
& \left.+\int_{0}^{1}|\mu-\alpha|\left|\Psi^{\prime}\left(\alpha \frac{a_{1}+a_{2}}{2}+(1-\alpha) a_{2}\right)\right| d \alpha\right] \\
\leq & \frac{a_{2}-a_{1}}{4}\left\{\left(\int_{0}^{1}|1-\lambda-\alpha| d \alpha\right)^{1-1 / r}\right. \\
& \times\left[\int_{0}^{1}|1-\lambda-\alpha|\left(\left(\frac{1+\alpha}{2}\right)^{s}\left|\Psi^{\prime}\left(a_{1}\right)\right|^{r}+\left(\frac{1-\alpha}{2}\right)^{s}\left|\Psi^{\prime}\left(a_{2}\right)\right|^{r}\right) d \alpha\right]^{1 / r} \\
& +\left(\int_{0}^{1}|\mu-\alpha| d \alpha\right)^{1-1 / r} \\
& \left.\times\left[\int_{0}^{1}|\mu-\alpha|\left(\left(\frac{\alpha}{2}\right)^{s}\left|\Psi^{\prime}\left(a_{1}\right)\right|^{r}+\left(\frac{2-\alpha}{2}\right)^{s}\left|\Psi^{\prime}\left(a_{2}\right)\right|^{r}\right) d t\right]^{1 / r}\right\}
\end{aligned}
$$


By direct calculation, we obtain

$$
\begin{aligned}
& \int_{0}^{1}|1-\lambda-\alpha|\left(\left(\frac{1+\alpha}{2}\right)^{s}\left|\Psi^{\prime}\left(a_{1}\right)\right|^{r}+\left(\frac{1-\alpha}{2}\right)^{s}\left|\Psi^{\prime}\left(a_{2}\right)\right|^{r}\right) d \alpha \\
= & \frac{\left|\Psi^{\prime}\left(a_{1}\right)\right|^{r}}{2^{s}} \int_{0}^{1}|1-\lambda-\alpha|(1+\alpha)^{s} d \alpha \\
& +\frac{\left|\Psi^{\prime}\left(a_{2}\right)\right|^{r}}{2^{s}} \int_{0}^{1}|1-\lambda-\alpha|(1-\alpha)^{s} d \alpha \\
= & \frac{\left|\Psi^{\prime}\left(a_{1}\right)\right|^{r}}{2^{s}(s+1)(s+2)}\left(2(2-\lambda)^{s+2}+(\lambda-1)\left(s+2^{s+2}+2\right)+2^{s+1} s \lambda-1\right) \\
& +\frac{\left|\Psi^{\prime}\left(a_{2}\right)\right|^{r}}{2^{s}(s+1)(s+2)}\left(2 \lambda^{s+2}+s(1-\lambda)-2 \lambda+1\right)
\end{aligned}
$$

Similarly, we have

$$
\begin{aligned}
& \int_{0}^{1}|\mu-\alpha|\left(\left(\frac{\alpha}{2}\right)^{s}\left|\Psi^{\prime}\left(a_{1}\right)\right|^{r}+\left(\frac{2-\alpha}{2}\right)^{s}\left|\Psi^{\prime}\left(a_{2}\right)\right|^{r}\right) d \alpha \\
= & \frac{\left|\Psi^{\prime}\left(a_{1}\right)\right|^{r}}{2^{s}} \int_{0}^{1}|\mu-\alpha| \alpha^{s} d t+\frac{\left|\Psi^{\prime}\left(a_{2}\right)\right|^{r}}{2^{s}} \int_{0}^{1}|\mu-\alpha|(2-\alpha)^{s} d \alpha \\
= & \frac{\left|\Psi^{\prime}\left(a_{1}\right)\right|^{r}}{2^{s}(s+1)(s+2)}\left(2 \mu^{s+2}+s(1-\mu)-2 \mu+1\right) \\
& +\frac{\left|\Psi^{\prime}\left(a_{2}\right)\right|^{r}}{2^{s}(s+1)(s+2)}\left(2(2-\mu)^{s+2}+(\mu-1)\left(s+2^{s+2}+2\right)+2^{s+1} s \mu-1\right) \\
\int_{0}^{1}|1-\lambda-\alpha| d \alpha=\frac{1}{2}\left(2 \lambda^{2}-2 \lambda+1\right) & \int_{0}^{1}|\mu-\alpha| d \alpha=\frac{1}{2}\left(2 \mu^{2}-2 \mu+1\right)
\end{aligned}
$$

Replace with the above four equalities into the inequality and the proof is finished.

Remark 2 In Theorem 2, if we take $s=1$, then Theorem 2 reduces to [14, Theorem 3.1]. Hence, the result in Theorem 2 is generalizations of the results of Xi et al. in [14, Theorem 3.1].

If taking $\lambda=\mu$ in (8), we derive the following corollary.

Corollary 5 Let $\Psi: J \subseteq \mathbb{R} \rightarrow \mathbb{R}$ be differentiable function on $J^{\circ}, a_{1}, a_{2} \in J$, $0 \leq \lambda \leq 1$, and $\Psi^{\prime} \in L\left[a_{1}, a_{2}\right]$. If $\left|\Psi^{\prime}(\varkappa)\right|^{r}$ is s-convex function on $\left[a_{1}, a_{2}\right]$ for some fixed $s \in(0,1]$, and $r \geq 1$, then

$$
\begin{aligned}
& \left|\frac{\lambda \Psi\left(a_{1}\right)+\lambda \Psi\left(a_{2}\right)}{2}+\frac{2-2 \lambda}{2} \Psi\left(\frac{a_{1}+a_{2}}{2}\right)-\frac{1}{a_{2}-a_{1}} \int_{a_{1}}^{a_{2}} \Psi(\varkappa) d \varkappa\right| \\
\leq & \frac{a_{2}-a_{1}}{8\left(2^{s-1}(s+1)(s+2)\right)^{1 / r}}\left(\left(2 \lambda^{2}-2 \lambda+1\right)\right)^{1-1 / r} \\
& \times\left\{\left[\left|\Psi^{\prime}\left(a_{1}\right)\right|^{r} E+\left|\Psi^{\prime}\left(a_{2}\right)\right|^{r} L\right]^{1 / r}+\left[\left|\Psi^{\prime}\left(a_{1}\right)\right|^{r} L+\left|\Psi^{\prime}\left(a_{2}\right)\right|^{r} E\right]^{1 / r}\right\}
\end{aligned}
$$


where $E$ and $L$ are like above.

If taking $\lambda=\mu=1 / 2,2 / 3,1 / 3$, respectively, in Theorem 2 , the following inequalities can be deduced.

Corollary 6 Let $s \in(0,1]$ and $\Psi: J \subseteq \mathbb{R} \rightarrow \mathbb{R}$ be differentiable function on $J^{\circ}$, $a_{1}, a_{2} \in J$ with $a_{1}<a_{2}$, and $\Psi^{\prime} \in L\left[a_{1}, a_{2}\right]$. If $\left|\Psi^{\prime}(\varkappa)\right|^{r}$ is s-convex function on $\left[a_{1}, a_{2}\right]$ for some fixed $s \in(0,1]$, and $r \geq 1$, then

$$
\begin{aligned}
& \left|\frac{1}{2}\left[\frac{\Psi\left(a_{1}\right)+\Psi\left(a_{2}\right)}{2}+\Psi\left(\frac{a_{1}+a_{2}}{2}\right)\right]-\frac{1}{a_{2}-a_{1}} \int_{a_{1}}^{a_{2}} \Psi(\varkappa) d \varkappa\right| \\
& \leq \frac{a_{2}-a_{1}}{8\left(2^{s-1}(s+1)(s+2)\right)^{1 / r}}\left(\frac{1}{2}\right)^{1-1 / r} \\
& \times\left\{\left[\left(\frac{3^{s+2}}{2^{s+1}}-\frac{2^{s+2}+s-2^{s+1} s+4}{2}\right)\left|\Psi^{\prime}\left(a_{1}\right)\right|^{r}\right.\right. \\
& \left.+\left(\frac{s}{2}+\frac{1}{2^{s+1}}\right)\left|\Psi^{\prime}\left(a_{2}\right)\right|^{r}\right]^{1 / r} \\
& +\left[\left(\frac{s}{2}+\frac{1}{2^{s+1}}\right)\left|\Psi^{\prime}\left(a_{1}\right)\right|^{r}\right. \\
& \left.\left.+\left(\frac{3^{s+2}}{2^{s+1}}-\frac{2^{s+2}+s-2^{s+1} s+4}{2}\right)\left|\Psi^{\prime}\left(a_{2}\right)\right|^{r}\right]^{1 / r}\right\} \\
& \left|\frac{1}{3}\left[\Psi\left(a_{1}\right)+\Psi\left(a_{2}\right)+\Psi\left(\frac{a_{1}+a_{2}}{2}\right)\right]-\frac{1}{a_{2}-a_{1}} \int_{a_{1}}^{a_{2}} \Psi(\varkappa) d \varkappa\right| \\
& \leq \frac{a_{2}-a_{1}}{8\left(2^{s-1}(s+1)(s+2)\right)^{1 / r}}\left(\frac{1}{2}\right)^{1-1 / r} \\
& \times\left\{\left[\left(\frac{2^{2 s+5}}{3^{s+2}}-\frac{2^{s+2}+s-2^{s+2} s+5}{3}\right)\left|\Psi^{\prime}\left(a_{1}\right)\right|^{r}\right.\right. \\
& \left.+\left(\frac{s-1}{3}+\frac{2^{s+3}}{3^{s+2}}\right)\left|\Psi^{\prime}\left(a_{2}\right)\right|^{r}\right]^{1 / r} \\
& +\left[\left(\frac{s-1}{3}+\frac{2^{s+3}}{3^{s+2}}\right)\left|\Psi^{\prime}\left(a_{1}\right)\right|^{r}\right. \\
& \left.\left.+\left(\frac{2^{2 s+5}}{3^{s+2}}-\frac{2^{s+2}+s-2^{s+2} s+5}{3}\right)\left|\Psi^{\prime}\left(a_{2}\right)\right|^{r}\right]^{1 / r}\right\},
\end{aligned}
$$




$$
\begin{aligned}
&\left|\frac{1}{6}\left[\Psi\left(a_{1}\right)+\Psi\left(a_{2}\right)+4 f\left(\frac{a_{1}+a_{2}}{2}\right)\right]-\frac{1}{a_{2}-a_{1}} \int_{a_{1}}^{a_{2}} \Psi(\varkappa) d \varkappa\right| \\
& \leq \frac{a_{2}-a_{1}}{8\left(2^{s-1}(s+1)(s+2)\right)^{1 / r}}\left(\frac{1}{2}\right)^{1-1 / r} \\
& \quad \times\left\{\left[\left(\frac{2 \times 5^{s+2}}{3^{s+2}}-\frac{2^{s+3}+2 s-2^{s+1} s+7}{3}\right)\left|\Psi^{\prime}\left(a_{1}\right)\right|^{r}\right.\right. \\
&\left.\quad+\left(\frac{2 s+1}{3}+\frac{2}{3^{s+2}}\right)\left|\Psi^{\prime}\left(a_{2}\right)\right|^{r}\right]^{1 / r} \\
&+\left[\left(\frac{2 s+1}{3}+\frac{2}{3^{s+2}}\right)\left|\Psi^{\prime}\left(a_{1}\right)\right|^{r}\right. \\
&\left.\left.\quad+\left(\frac{2 \times 5^{s+2}}{3^{s+2}}-\frac{2^{s+3}+2 s-2^{s+1} s+7}{3}\right)\left|\Psi^{\prime}\left(a_{2}\right)\right|^{r}\right]^{1 / r}\right\} .
\end{aligned}
$$

Remark 3 If setting $s=1$ in above Corollary, then we obtain the inequalities in [14, pp. 6 and 7$]$.

Remark 4 If setting $s=1$ and $r=1$ in above Corollary, then we obtain the inequalities in [14, (3.7)]. Hence, the results in above Corollary are generalizations of the results of $X i$ and $Q i$ in [14].

\section{Applications}

Let

$$
\begin{aligned}
A\left(a_{1}, a_{2}\right) & =\frac{a_{1}+a_{2}}{2}, L\left(a_{1}, a_{2}\right)=\frac{a_{2}-a_{1}}{\ln a_{2}-\ln a_{1}} \quad\left(a_{1} \neq a_{2}\right), \\
L_{p}\left(a_{1}, a_{2}\right) & =\left(\frac{a_{2}^{p+1}-a_{1}^{p+1}}{(p+1)\left(a_{2}-a_{1}\right)}\right)^{1 / p}, a_{1} \neq a_{2}, p \in \mathbb{R}, p \neq-1,0
\end{aligned}
$$

be the arithmetic mean, logarithmic mean, generalized logarithmic mean for $a_{1}, a_{2}>$ 0 respectively.

Propertie 1 Let $g: \mathbb{R} \rightarrow \mathbb{R}_{+}$be a non-negative convex function on $\mathbb{R}$. Then $g^{s}(\varkappa)$ is s-convex on $J$, for some fixed $s \in(0,1)$ (see [1]). 
Proposition 1 Let $s \in(0,1], a_{1}, a_{2}>0,1 / p+1 / r=1,0 \leq \lambda, \mu \leq 1$, then

$$
\begin{aligned}
& \left|\frac{\lambda u^{s}+\mu v^{s}}{2}+\frac{2-\lambda-\mu}{2} A^{s}\left(a_{1}, a_{2}\right)-L_{s}^{s}\left(a_{1}, a_{2}\right)\right| \\
\leq & \frac{a_{2}-a_{1}}{4}\left(\frac{(1-\lambda)^{p+1}+\lambda^{p+1}}{p+1}\right)^{\frac{1}{p}}\left(\frac{s\left(2^{s+1}-1\right) a_{1}^{r(s-1)}}{2^{s}(s+1)}+\frac{s v^{r(s-1)}}{2^{s}(s+1)}\right)^{\frac{1}{r}} \\
& +\frac{a_{2}-a_{1}}{4}\left(\frac{\mu^{p+1}+(1-\mu)^{p+1}}{p+1}\right)^{\frac{1}{p}}\left(\frac{s a_{1}^{r(s-1)}}{2^{s}(s+1)}+\frac{s\left(2^{s+1}-1\right) a_{2}^{r(s-1)}}{2^{s}(s+1)}\right)^{\frac{1}{r}}
\end{aligned}
$$

In particular, when $\lambda=\mu=1$, we have

$$
\begin{aligned}
& \left|A\left(a_{1}^{s}, a_{2}^{s}\right)-L_{s}^{s}\left(a_{1}, a_{2}\right)\right| \\
\leq & \frac{a_{2}-a_{1}}{4}\left(\frac{1}{p+1}\right)^{\frac{1}{p}}\left(\frac{s\left(2^{s+1}-1\right) a_{1}^{r(s-1)}}{2^{s}(s+1)}+\frac{s v^{r(s-1)}}{2^{s}(s+1)}\right)^{\frac{1}{r}} \\
& +\frac{a_{2}-a_{1}}{4}\left(\frac{1}{p+1}\right)^{\frac{1}{p}}\left(\frac{s a_{1}^{r(s-1)}}{2^{s}(s+1)}+\frac{s\left(2^{s+1}-1\right) a_{2}^{r(s-1)}}{2^{s}(s+1)}\right)^{\frac{1}{r}}
\end{aligned}
$$

Proof. The claim follows from Theorem 1 applied to $s$-convex in the second sense mapping $\Psi(\varkappa)=\varkappa^{s}$.

Remark 5 In Proposition 1, if we take $\lambda=\mu=1 / 3$, then Proposition 1 reduces to [12, pp. 2198]. Hence, the results in Proposition 1 are generalizations of the results of Sarikaya et al. in [12].

Proposition 2 Let $s \in(0,1], a_{1}, a_{2}>0, r \geq 1,0 \leq \lambda, \mu \leq 1$, then

$$
\begin{aligned}
& \left|\frac{\lambda a_{1}^{s}+\mu a_{2}^{s}}{2}+\frac{2-\lambda-\mu}{2} A^{s}\left(a_{1}, a_{2}\right)-L_{s}^{s}\left(a_{1}, a_{2}\right)\right| \\
\leq & \frac{s\left(a_{2}-a_{1}\right)}{8}\left(\frac{1}{2^{s-1}(s+1)(s+2)}\right)^{1 / r} \\
& \times\left\{\left(\left(2 \lambda^{2}-2 \lambda+1\right)\right)^{1-1 / r} \times\left[a_{1}^{r(s-1)} E+a_{2}^{r(s-1)} L\right]^{1 / r}\right. \\
& \left.+\left(\left(2 \mu^{2}-2 \mu+1\right)\right)^{1-1 / r} \times\left[a_{1}^{r(s-1)} J+a_{2}^{r(s-1)} F\right]^{1 / r}\right\}
\end{aligned}
$$

In particular, when $\lambda=\mu=1$, we have

$$
\begin{aligned}
& \left|A\left(a_{1}^{s}, a_{2}^{s}\right)-L_{s}^{s}\left(a_{1}, a_{2}\right)\right| \\
\leq & \frac{s\left(a_{2}-a_{1}\right)}{8}\left(\frac{1}{2^{s-1}(s+1)(s+2)}\right)^{1 / r} \times\left\{\left[a_{1}^{r(s-1)}\left(1+s 2^{s+1}\right)+a_{2}^{r(s-1)}\right]^{1 / r}\right. \\
+ & {\left.\left[a_{1}^{r(s-1)}+a_{2}^{r(s-1)}\left(1+s 2^{s+1}\right)\right]^{1 / r}\right\} }
\end{aligned}
$$


Moreover, when $r=1$, we have

$$
\left|A\left(a_{1}^{s}, a_{2}^{s}\right)-L_{s}^{s}\left(a_{1}, a_{2}\right)\right| \leq \frac{s^{2}\left(1+s 2^{s}\right)\left(a_{2}-a_{1}\right)}{2^{s}(s+1)(s+2)} \times A\left(a_{1}^{s-1}, a_{2}^{s-1}\right)
$$

Proof. The claim follows from Theorem 2 applied to $s$-convex in the second sense mapping $\Psi(\varkappa)=\varkappa^{s}$.

Proposition 3 Let $s \in(0,1], a_{1}, a_{2}>0, r \geq 1,0 \leq \lambda, \mu \leq 1$, then

(9)

$$
\begin{aligned}
& \left|\frac{\lambda a^{-s}+\mu v^{-s}}{2}+\frac{2-\lambda-\mu}{2} A^{-s}\left(a_{1}, a_{2}\right)-L_{-s}^{-s}\left(a_{1}, a_{2}\right)\right| \\
\leq & \frac{s\left(a_{2}-a_{1}\right)}{8}\left(\frac{1}{2^{s-1}(s+1)(s+2)}\right)^{1 / r} \\
& \times\left\{\left(\left(2 \lambda^{2}-2 \lambda+1\right)\right)^{1-1 / r} \times\left[\left|a_{1}^{-r(s+1)}\right| E+a_{2}^{-r(s+1)} L\right]^{1 / r}\right. \\
& \left.+\left(\left(2 \mu^{2}-2 \mu+1\right)\right)^{1-1 / r} \times\left[a_{1}^{-r(s+1)} J+a_{2}^{-r(s+1)} F\right]^{1 / r}\right\}
\end{aligned}
$$

In particular, when $\lambda=\mu=1 / 3$, we have

$$
\begin{aligned}
& \left|\frac{1}{3} A\left(a_{1}^{-s}, a_{2}^{-s}\right)+\frac{2}{3} A^{-s}\left(a_{1}, a_{2}\right)-L_{-s}^{-s}\left(a_{1}, a_{2}\right)\right| \\
\leq & \frac{s\left(a_{2}-a_{1}\right)}{8}\left(\frac{1}{2^{s-1}(s+1)(s+2)}\right)^{1 / r}\left(\frac{5}{9}\right)^{1-1 / r} \\
& \times\left\{\left[\left(\frac{2 \times 5^{s+2}}{3^{s+2}}-\frac{2^{s+3}+2 s-2^{s+1} s+7}{3}\right)\left|a_{1}^{-r(s+1)}\right|\right.\right. \\
& \left.+\left(\frac{2 s+1}{3}+\frac{2}{3^{s+2}}\right)\left|a_{2}^{-r(s+1)}\right|\right]^{1 / r} \\
& +\left[\left(\frac{2 s+1}{3}+\frac{2}{3^{s+2}}\right)\left|a_{1}^{-r(s+1)}\right|\right. \\
& \left.\left.+\left(\frac{2 \times 5^{s+2}}{3^{s+2}}-\frac{2^{s+3}+2 s-2^{s+1} s+7}{3}\right)\left|a_{2}^{-r(s+1)}\right|\right]^{1 / r}\right\}
\end{aligned}
$$

Moreover, when $s=r=1$, we obtain

$$
\left|\frac{1}{3} A\left(a_{1}^{-1}, a_{2}^{-1}\right)+\frac{2}{3} A^{-1}\left(a_{1}, a_{2}\right)-L_{-1}^{-1}\left(a_{1}, a_{2}\right)\right| \leq\left(a_{2}-a_{1}\right) \frac{5}{36} A\left(a_{1}^{-2}, a_{2}^{-2}\right),
$$

that it is the inequality in [12, pp. 2199, line 2].

Proof. The claim follows from Theorem 2 applied to $s$-convex in the second sense mapping $\Psi(\varkappa)=\frac{1}{\varkappa^{s}}$. 
Remark 6 If we take $\lambda=\mu=1 / 3$ in Proposition 3, then inequality (9) reduces to [12, pp. 2199]. Hence, the results in Proposition 3 are generalizations of the results of Sarikaya et al. in [12].

\section{References}

[1] M. Alomari, M. Darus, S. S. Dragomir, New inequalities of Simpson's type for s-convex functions with applications, RGMIA Res. Rep. Coll., Article 9, vol. 12, no.4, 2009.

[2] G. A. Anastassiou, Taylor Widder representation formulae and Ostrowski, Grüss, integral means and Csiszar type inequalities, Comp\&Math Appl., vol. 54, 2007, 9-23.

[3] M. Bessenyei, Z. Páles, Characterization of convexity via Hadamard's inequality, Math. Inequal. Appl., vol. 9, 2006, 53-62.

[4] S. S. Dragomir, R. P. Agarwal, P. Cerone, On Simpson's inequality and applications, Journal of Ineq. and Appl., vol. 5, 2000, 533-579.

[5] S. S. Dragomir, C. E. M. Pearce, Selected topics on Hermite-Hadamard inequalities and applications, RGMIA monographs, Victoria University, 2000.

[6] J. Hadamard, Étude sur les propriétés des fonctions entières en particulier d'une fonction considérée par Riemann, J. Math. Pures Appl., vol. 58, 1893, 171-215.

[7] H. Hudzik, L. Maligranda, Some remarks on s-convex functions, Aequationes Math., vol. 48, 1994, 100-111.

[8] D. S. Mitrinović, I. B. Lacković, Hermite and convexity, Aequationes Math., vol. 28, 1985, 229-232.

[9] D. S. Mitrinovic, J. E. Pecaric, A. M. Fink, Classical and New Inequalities in Analysis, Kluwer Academic Publishers, 1993.

[10] C. P. Niculescu, L. Persson, Convex Functions and their Applications, A Contemporary Approach, in: CMS Books in Mathematics, 23, Springer-Verlag, New York, 2006.

[11] M. Z. Sarikaya, E. Set, M. E. Özdemir, On new inequalities of Simpson's type for convex functions, RGMIA Res. Rep. Coll., Article 2, vol. 13, no. 2, 2010.

[12] M. Z. Sarikaya, E. Set, M. E. Özdemir, On new inequalities of Simpson's type for s-convex functions, Comp\&Math Appl, vol. 60, 2010, 2191-2199.

[13] S. Wu, L. Debnath, Inequalities for convex sequences and their applications, Comp\&Math Appl., vol. 54, 2007, 525-534. 
[14] B. Y. Xi, F. Qi, Some Integral Inequalities of Hermite-Hadamard Type for Convex Functions with Applications to Means, Journal of Function Spaces and Appl., Article ID 980438, vol. 2012, doi: 10.1155/2012/980438.

\section{Musa Çakmak}

Hatay Mustafa Kemal University

Reyhanl Vocational School of Social Sciences

Hatay, Turkey

e-mail: enkucukcakmak@gmail.com

\section{Mevlüt Tunç}

Saraykent M. 273. S.

Antakya, Hatay, Turkey

e-mail:mevluttttunc@gmail.com 